\title{
Effect of glyphosate-based herbicide on early life stages of Java medaka (Oryzias javanicus): a potential tropical test fish
}

\begin{abstract}
Glyphosate is globally a widely used herbicide, yet there is little information on their toxicity to marine fishes. Java medaka, a small tropical fish native to coastal areas in several Southeast Asian countries, is viewed as a suitable candidate for toxicity test and thus was used for this study. Java medaka adults were cultured in the laboratory and the fertilized eggs of the F2 generation were exposed to different concentrations of glyphosate-based herbicide $(100,200,300,400$ and $500 \mathrm{ppm})$ until they hatched. The survival and hatching rates of the embryos, changes in the heart rate and morphological impairments were recorded. Generally, survival and hatching percentage decreased as glyphosate concentration increased. Absence of pectoral fin(s) and cornea, permanently bent tail, irregular shaped abdomen, and cell disruption in the fin, head and abdomen are among the common teratogenic effects observed. Furthermore, risk factor also increased with the increased in glyphosate concentrations.
\end{abstract}

Keyword: Glyphosate; Java medaka; Toxicity; Teratogenesis 